\title{
On the Existence of a Unique Solution for a Class of Fractional Differential Inclusions in a Hilbert Space
}

\author{
Mikhail Kamenskii ${ }^{1}$, Valeri Obukhovskii ${ }^{2}$, Garik Petrosyan ${ }^{3}$ and Jen-Chih Yao ${ }^{4, *}$ \\ 1 Faculty of Mathematics, Voronezh State University, Voronezh 394018, Russia; mikhailkamenski@mail.ru \\ 2 Faculty of Physics and Mathematics, Voronezh State Pedagogical University, Voronezh 394043, Russia; \\ valerio-ob2000@mail.ru \\ 3 Research Center of Voronezh State University of Engineering Technologies and Faculty of Physics and \\ Mathematics, Voronezh State Pedagogical University, Voronezh 394043, Russia; garikpetrosyan@yandex.ru \\ 4 Research Center for Interneural Computing, China Medical University Hospital, China Medical University, \\ Taichung 40402, Taiwan \\ * Correspondence: yaojc@mail.cmu.edu.tw
}

Citation: Kamenskii, M.;

Obukhovskii, V.; Petrosyan, G.; Yao, J.-C. On the Existence of a Unique Solution for a Class of Fractional Differential Inclusions in a Hilbert Space. Mathematics 2021, 9, 136. https://dx.doi.org/10.3390/math902 0136

Received: 14 December 2020 Accepted: 6 January 2021

Published: 10 January 2021

Publisher's Note: MDPI stays neutral with regard to jurisdictional claims in published maps and institutional affiliations.

Copyright: () 2021 by the authors. Licensee MDPI, Basel, Switzerland. This article is an open access article distributed under the terms and conditions of the Creative Commons Attribution (CC BY) license (https: / / creativecommons.org/ licenses/by/4.0/).

\begin{abstract}
We obtained results on the existence and uniqueness of a mild solution for a fractional-order semi-linear differential inclusion in a Hilbert space whose right-hand side contains an unbounded linear monotone operator and a Carathéodory-type multivalued nonlinearity satisfying some monotonicity condition in the phase variables. We used the Yosida approximations of the linear part of the inclusion, the method of a priori estimates of solutions, and the topological degree method for condensing vector fields. As an example, we considered the existence and uniqueness of a solution to the Cauchy problem for a system governed by a perturbed subdifferential inclusion of a fractional diffusion type.
\end{abstract}

Keywords: fractional differential inclusion; semi-linear differential inclusion; Cauchy problem; monotonicity condition; existence; uniqueness; a priori estimate; Yosida approximation; condensing operator; topological degree

MSC: Primary: 34QA08; Secondary: 34B15, 34C12, 47H04, 47H05, 47H08, 47H11

\section{Introduction}

Recent years have seen a wide spread of fractional analysis as well as the theory of fractional-order differential equations and inclusions in contemporary mathematics. The increasing interest in this subject is explained by its numerous applications in various branches of applied mathematics, physics, engineering, biology, economics, and other sciences (see, e.g., the monographs of [1-3]). A number of various approaches to the solving of boundary value problems for differential equations and inclusions in the case of fractional order $q \in(0,1)$ have been widely described in the literature (see [4-19] and references therein).

It is well known that, beginning with the classical Cauchy-Picard theorem, as the usual assumption providing the existence and uniqueness of the solution to the initial value problem

$$
\begin{gathered}
x^{\prime}(t)=f(t, x(t)), \\
x(0)=x_{0},
\end{gathered}
$$

the Lipschitz condition of the function $f$ in the space variable $x$ is considered. However, for a large class of such problems, especially in the case of an infinite-dimensional phase space, this condition looks rather onerous. That is why, in a large number of works (see, for example, Refs. [20-24]), the Lipschitz condition is replaced with a certain type of 
monotonicity condition of $f$ in $x$. For example, in the case of a Hilbert space $H$ with the inner product $\langle\cdot, \cdot\rangle$, this condition can take the form

$$
\left\langle f\left(t, x_{1}\right)-f\left(t, x_{2}\right), x_{1}-x_{2}\right\rangle \leq a\left\|x_{1}-x_{2}\right\|^{2}, \quad \forall x_{1}, x_{2} \in H
$$

for some $a>0$. Notice that the existence and uniqueness results also remain true in the case of differential inclusion, i.e., when the right-hand side $f$ is a multivalued map.

To the best of our knowledge, for fractional-order differential equations and inclusions (see, e.g., [1-3] and references therein), the results of such a type have not been obtained up to the present time. In our opinion, the property of the Caputo fractional derivative

$$
{ }^{C} D_{0}^{q}\|x(t)\| \leq\left\langle x(t),{ }^{C} D_{0}^{q} x(t)\right\rangle
$$

of a function $x(\cdot)$ with the values in a Hilbert space, which was recently proved in the works [25-27], opens up some opportunities in this direction.

In the present paper, we obtain results on the existence and uniqueness of a mild solution to the Cauchy problem for a fractional-order semi-linear differential inclusion in a Hilbert space whose right-hand side contains an unbounded linear monotone operator and a multivalued nonlinearity satisfying a condition of type (3). The paper is organized in the following way. In the next section, we recall necessary information from fractional calculus, the theory of measures of noncompactness, and the topological degree theory for condensing multivalued maps. In the third section, we formulate the problem and develop some approximation results based on the Yosida approximations of the linear part of the inclusion. This allows us to obtain the result (Theorem 1) about the a priori estimate for a mild solution of the problem. This theorem is used to get the result (Theorem 2) on the existence of a mild solution to the problem on an arbitrary bounded interval. Further, the main result of the fourth section is Theorem 3 on the uniqueness of a mild solution to the problem. Finally, in the fifth section, we present the existence and uniqueness of a solution to the Cauchy problem for a system governed by a perturbed subdifferential inclusion of a fractional diffusion type as an example.

\section{Preliminaries}

\subsection{Fractional Derivative}

In this section, we will recall some notions and definitions that we will need in the following sections (details can be found, e.g., in [1-3,28]).

Let $E$ be a real Banach space.

Definition 1. The Riemann-Liouville fractional derivative of the order $q \in(0,1)$ of a continuous function $g:[0, a] \rightarrow E$ is the function $D_{0}^{q} g$ of the following form:

$$
D_{0}^{q} g(t)=\frac{1}{\Gamma(1-q)} \frac{d}{d t} \int_{0}^{t}(t-s)^{-q} g(s) d s,
$$

provided that the right-hand side of this equality is well defined.

Here, $\Gamma$ is the Euler gamma-function

$$
\Gamma(r)=\int_{0}^{\infty} s^{r-1} e^{-s} d s .
$$

Definition 2. The Caputo fractional derivative of the order $q \in(0,1)$ of a continuous function $g:[0, a] \rightarrow E$ is the function ${ }^{C} D_{0}^{q} g$ defined in the following way:

$$
{ }^{C} D_{0}^{q} g(t)=\left(D^{q}(g(\cdot)-g(0))\right)(t),
$$

provided that the right-hand side of this equality is well defined. 
Definition 3. A function of the form

$$
E_{q, \beta}(z)=\sum_{n=0}^{\infty} \frac{z^{n}}{\Gamma(q n+\beta)}, \quad q, \beta>0, z \in \mathbb{C},
$$

is called the Mittag-Leffler function.

The Mittag-Leffler function has the following asymptotic representation as $z \rightarrow \infty$ (see, e.g., $[28,29])$ :

$$
E_{q, \beta}(z)= \begin{cases}\frac{1}{q} z^{\frac{1-\beta}{q}} e^{z^{\frac{1}{q}}}-\sum_{n=1}^{N-1} \frac{z^{-n}}{\Gamma(\beta-q n)}+O\left(|z|^{-N}\right), & |\arg z| \leq \frac{1}{2} \pi q, \\ -\sum_{n=1}^{N-1} \frac{z^{-n}}{\Gamma(\beta-q n)}+O\left(|z|^{-N}\right), & |\arg (-z)| \leq\left(1-\frac{1}{2} q\right) \pi .\end{cases}
$$

Denote $E_{q, 1}$ by $E_{q}$. Notice that the second of the above formula implies that in the case where $z=\tau<0$ and $0<q<1$, we have

$$
E_{q}(\tau) \rightarrow 0 \text { as } \tau \rightarrow-\infty .
$$

Notice that from the relations (see, e.g., [30]):

$$
E_{q}(-z)=\int_{0}^{\infty} \xi_{q}(\theta) e^{-z \theta} d \theta
$$

and

$$
E_{q, q}(-z)=\int_{0}^{\infty} q \theta \xi_{q}(\theta) e^{-z \theta} d \theta
$$

where

$$
\begin{gathered}
\xi_{q}(\theta)=\frac{1}{q} \theta^{-1-\frac{1}{q}} \Psi_{q}\left(\theta^{-1 / q}\right), \\
\Psi_{q}(\theta)=\frac{1}{\pi} \sum_{n=1}^{\infty}(-1)^{n-1} \theta^{-q n-1} \frac{\Gamma(n q+1)}{n !} \sin (n \pi q), \theta \in \mathbb{R}_{+},
\end{gathered}
$$

it follows that

$$
E_{q}(\tau)>0, E_{q, q}(\tau)>0 \text { for } \tau<0
$$

$\operatorname{Remark} 1$ (See, e.g., [31]). $\xi_{q}(\theta) \geq 0, \int_{0}^{\infty} \xi_{q}(\theta) d \theta=1, \int_{0}^{\infty} \theta \xi_{q}(\theta) d \theta=\frac{1}{\Gamma(q+1)}$.

Consider a scalar equation of the form

$$
{ }^{C} D^{q} x(t)=\lambda x(t)+f(t), \quad t \in[0, T],
$$

with the initial condition

$$
x(0)=x_{0},
$$

where $\lambda \in \mathbb{R}, f:[0, T] \rightarrow \mathbb{R}$ is a continuous function. By a solution of this problem, we mean a continuous function $x:[0, T] \rightarrow \mathbb{R}$ satisfying condition (11) whose fractional derivative ${ }^{C} D^{q} x$ is also continuous and satisfies Equation (10). It is known (see [1], Example 4.9) that the unique solution of this equation has the form

$$
x(t)=E_{q}\left(\lambda t^{q}\right) x_{0}+\int_{0}^{t}(t-s)^{q-1} E_{q, q}\left(\lambda(t-s)^{q}\right) f(s) d s .
$$

We will need the following auxiliary assertion, which is an analogue of the known Gronwall lemma on integral inequalities. 
Lemma 1. Let a bounded measurable function $z:[0, T] \rightarrow \mathbb{R}$ such that ${ }^{C} D_{0}^{q} z$ is a continuous function and

$$
{ }^{C} D_{0}^{q} z(t) \leq \lambda z(t)+l(t), \quad t \in[0, T],
$$

where $l:[0, T] \rightarrow \mathbb{R}$ is a bounded measurable function. Then,

$$
z(t) \leq E_{q}\left(\lambda t^{q}\right) z(0)+\int_{0}^{t}(t-s)^{q-1} E_{q, q}\left(\lambda(t-s)^{q}\right) l(s) d s .
$$

Proof. Consider a scalar equation

$$
{ }^{C} D_{0}^{q} y(t)=\lambda y(t)+l(t), \quad t \in[0, T],
$$

with the initial condition $y(0)=z(0)$. From inequality (13) and Equation (14), we have

$$
{ }^{C} D_{0}^{q}(z(t)-y(t)) \leq \lambda(z(t)-y(t)) .
$$

There exists a non-negative function $v:[0, T] \rightarrow \mathbb{R}$ such that

$$
{ }^{C} D_{0}^{q}(z(t)-y(t))=\lambda(z(t)-y(t))-v(t) .
$$

The solution to the last equation is the following non-negative function

$$
y(t)-z(t)=\int_{0}^{t}(t-s)^{q-1} E_{q, q}\left(\lambda(t-s)^{q}\right) v(s) d s .
$$

Thus, $z(t) \leq y(t)$ because

$$
y(t)=E_{q}\left(\lambda t^{q}\right) y(0)+\int_{0}^{t}(t-s)^{q-1} E_{q, q}\left(\lambda(t-s)^{q}\right) l(s) d s,
$$

and $y(0)=z(0)$; we finally get the inequality

$$
z(t) \leq E_{q}\left(\lambda t^{q}\right) z(0)+\int_{0}^{t}(t-s)^{q-1} E_{q, q}\left(\lambda(t-s)^{q}\right) l(s) d s .
$$

\subsection{Measures of Noncompactness and Condensing Multivalued Maps}

Let us recall some notions and facts (details can be found, for example, in [32-36]).

Let $\mathcal{E}$ be a Banach space. We introduce the following notation:

- $\quad \operatorname{Pb}(\mathcal{E})=\{A \subseteq \mathcal{E}: A \neq \varnothing$ is bounded $\} ;$

- $\operatorname{Pv}(\mathcal{E})=\{A \in \operatorname{Pb}(\mathcal{E}): A$ is convex $\}$

- $K(\mathcal{E})=\{A \in P b(\mathcal{E}): A$ is compact $\}$;

- $K v(\mathcal{E})=\operatorname{Pv}(\mathcal{E}) \cap K(\mathcal{E})$.

Definition 4. Let $(\mathcal{A}, \geq)$ be a partially ordered set. A function $\beta: P b(\mathcal{E}) \rightarrow \mathcal{A}$ is called the measure of noncompactness $(M N C)$ in $\mathcal{E}$ if, for each $\Omega \in P b(\mathcal{E})$, we have:

$$
\beta(\overline{\mathrm{co}} \Omega)=\beta(\Omega),
$$

where $\overline{\mathrm{co}} \Omega$ denotes the closure of the convex hull of $\Omega$.

A measure of noncompactness $\beta$ is called:

(1) monotone if for, each $\Omega_{0}, \Omega_{1} \in P b(\mathcal{E}), \Omega_{0} \subseteq \Omega_{1}$ implies $\beta\left(\Omega_{0}\right) \leq \beta\left(\Omega_{1}\right)$;

(2) nonsingular if, for each $a \in \mathcal{E}$ and each $\Omega \in P b(\mathcal{E})$, we have $\beta(\{a\} \cup \Omega)=\beta(\Omega)$.

If $\mathcal{A}$ is a cone in a Banach space, the MNC $\beta$ is called:

(1) regular if $\beta(\Omega)=0$ is equivalent to the relative compactness of $\Omega \in P b(\mathcal{E})$; 
(2) real if $\mathcal{A}$ is the set of all real numbers $\mathbb{R}$ with the natural ordering;

(3) algebraically semiadditive if $\beta\left(\Omega_{0}+\Omega_{1}\right) \leq \beta\left(\Omega_{0}\right)+\beta\left(\Omega_{1}\right)$ for every $\Omega_{0}, \Omega_{1} \in P b(\mathcal{E})$.

It should be mentioned that the Hausdorff MNC obeys all of the above properties. Another example can be presented by the following measures of noncompactness defined on $\operatorname{Pb}(C([0, a] ; E))$, where $C([0, a] ; E)$ is the space of continuous functions with the values in a separable Banach space $E$ :

(i) the modulus of fiber noncompactness

$$
\varphi(\Omega)=\sup _{t \in[0, a]} e^{-p t} \chi_{E}(\Omega(t)),
$$

where $p>0, \chi_{E}$ is the Hausdorff MNC in $E$ and $\Omega(t)=\{y(t): y \in \Omega\}$;

(ii) the modulus of equicontinuity, defined as

$$
\bmod _{C}(\Omega)=\lim _{\delta \rightarrow 0} \sup _{y \in \Omega} \max _{\left|t_{1}-t_{2}\right| \leq \delta}\left\|y\left(t_{1}\right)-y\left(t_{2}\right)\right\| .
$$

Notice that these MNCs satisfy all above-mentioned properties except regularity. To obtain a regular MNC in the space $C([0, a] ; E)$, we can consider the MNC

$$
v(\Omega)=\left(\varphi(\Omega), \bmod _{C}(\Omega)\right)
$$

with the values in the cone $\mathbb{R}^{2}$ with the natural partial order.

Definition 5. Let $X \subseteq \mathcal{E}$ be a closed subset; a multivalued map (multimap) $\mathcal{F}: X \rightarrow K(\mathcal{E})$ is called upper semicontinuous (u.s.c.) if the pre-image

$$
\mathcal{F}^{-1}(V)=\{x \in X: \mathcal{F}(x) \subset V\}
$$

of each open set $V \subset \mathcal{E}$ is open in $X$.

Definition 6. A u.s.c. multimap $\mathcal{F}: X \rightarrow K(\mathcal{E})$ is called condensing with respect to an $M N C \beta$ (or $\beta$-condensing) if, for every bounded set $\Omega \subseteq X$ that is not relatively compact, we have

$$
\beta(\mathcal{F}(\Omega)) \nsupseteq \beta(\Omega) .
$$

More generally, given a metric space $\Lambda$ of parameters, we will say that a u.s.c. multimap $\Gamma: \Lambda \times X \rightarrow K(\mathcal{E})$ is a condensing family with respect to an MNC $\beta$ (or $\beta$-condensing family) if, for every bounded set $\Omega \subseteq X$ that is not relatively compact, we have

$$
\beta(\Gamma(\Lambda \times \Omega)) \nsupseteq \beta(\Omega) .
$$

Let $V \subset \mathcal{E}$ be a bounded open set, $\beta$ a monotone nonsingular MNC in $\mathcal{E}$, and $\mathcal{F}$ : $\bar{V} \rightarrow K v(\mathcal{E})$ a $\beta$-condensing multimap such that $x \notin \mathcal{F}(x)$ for all $x \in \partial V$, where $\bar{V}$ and $\partial V$ denote the closure and the boundary of the set $V$.

In such a setting, the topological degree

$$
\operatorname{deg}(i-\mathcal{F}, \bar{V})
$$

of the corresponding vector multifield $i-\mathcal{F}$ satisfying the standard properties is defined, where $i$ is the identity map on $\mathcal{E}$. In particular, the condition

$$
\operatorname{deg}(i-\mathcal{F}, \bar{V}) \neq 0
$$

implies that the fixed-point set Fix $\mathcal{F}=\{x: x \in \mathcal{F}(x)\}$ is a nonempty subset of $V$.

To describe the next property, let us introduce the following notion. 
Definition 7. Suppose that $\beta$-condensing multimaps $\mathcal{F}_{0}, \mathcal{F}_{1}: \bar{V} \rightarrow \operatorname{Kv}(\mathcal{E})$ have no fixed points on the boundary $\partial V$ and there exists a $\beta$-condensing family $\mathcal{H}:[0,1] \times \bar{V} \rightarrow K(\mathcal{E})$ such that:

(i) $x \notin \mathcal{H}(\lambda, x)$ for all $(\lambda, x) \in[0,1] \times \partial V$;

(ii) $\mathcal{H}(0, \cdot)=\mathcal{F}_{0} ; \mathcal{H}(1, \cdot)=\mathcal{F}_{1}$.

Then, the multifields $\Phi_{0}=i-\mathcal{F}_{0}$ and $\Phi_{1}=i-\mathcal{F}_{1}$ are called homotopic:

$$
\Phi_{0} \sim \Phi_{1} .
$$

The homotopy invariance property of the topological degree asserts that if $\Phi_{0} \sim \Phi_{1}$, then $\operatorname{deg}\left(i-\mathcal{F}_{0}, \bar{V}\right)=\operatorname{deg}\left(i-\mathcal{F}_{1}, \bar{V}\right)$.

Let us also mention the following property of the topological degree, which we will need in later.

The normalization property: If $\mathcal{F}(x) \equiv A \in K(\mathcal{E})$, then

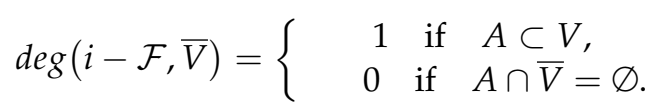

\section{Existence of a Solution}

Let $H$ be a separable Hilbert space. We will consider the Cauchy problem for a semi-linear fractional-order differential inclusion in $H$ :

$$
\begin{gathered}
{ }^{C} D_{0}^{q} x(t) \in A x(t)+F(t, x(t)), \quad t \in[0, T], \\
x(0)=x_{0},
\end{gathered}
$$

where $0<q<1$, and the linear operator $A$ satisfies the following condition:

(A) $A: D(A) \subseteq H \rightarrow H$ is a linear closed (not necessarily bounded) operator generating a bounded $C_{0}$-semigroup $\left\{U_{A}(t)\right\}_{t \geq 0}$ of linear operators in $H$ such that

$$
\langle A x, x\rangle \leq-d\|x\|^{2}, \quad \forall x \in D(A),
$$

for some $d>0$. ditions:

It will be assumed that the multimap $F:[0, T] \times H \rightarrow K v(H)$ obeys the following con-

$(F 1)$ The multifunction $F(\cdot, x):[0, T] \rightarrow K v(H)$ admits a measurable selection for each $T>0$ and $x \in H$, i.e., there exists a measurable function $f:[0, T] \rightarrow H$ such that $f(t) \in F(t, x)$ for a.e. $t \in[0, T]$;

(F2) The multimap $F(t, \cdot): H \rightarrow K v(H)$ is u.s.c. for each $T>0$ and for a.e. $t \in[0, T]$;

(F3) For each $R>0$ and $T>0$, there exists a function $\omega_{R} \in L^{\infty}[0, T]$ such that $x \in H$, $\|x\| \leq R$ implies

$$
\|F(t, x)\| \leq \omega_{R}(t) \text { for a.e. } t \in[0, T] ;
$$

(F4) For each $T>0$, there exists $\kappa \in L^{\infty}[0, T]$ such that, for every bounded set $\Omega \subset H$, it holds that:

$$
\chi(t, \Omega)) \leq \kappa(t) \chi(\Omega),
$$

where $\chi$ denotes the Hausdorff MNC in the space $H$.

(F5) for each $x, \widetilde{x} \in H$ and $y \in F(t, x), \widetilde{y} \in F(t, \widetilde{x}), t \in[0, T]$ it holds:

$$
\langle y-\tilde{y}, x-\tilde{x}\rangle \leq a\|x-\tilde{x}\|^{2} .
$$

From conditions $(F 1)-(F 3)$, it follows that for each $T>0$, the superposition multioperator $\mathcal{P}_{F}: C([0, T] ; H) \rightarrow P\left(L^{\infty}((0, T) ; H)\right)$ is defined by the formula

$$
\mathcal{P}_{F}(x)=\left\{f \in L^{\infty}((0, T) ; H): f(s) \in F(s, x(s)) \text { for a.e. } s \in[0, T]\right\}
$$


(see, for example, [32,33]).

Let us recall (see, for example, [4-19]) that a mild solution to problems (15) and (16) is a function $x \in C([0, T], H)$ of the form

$$
x(t)=\mathcal{G}_{A}(t) x_{0}+\int_{0}^{t}(t-s)^{q-1} \mathcal{T}_{A}(t-s) f(s) d s,
$$

where

$$
\mathcal{G}_{A}(t)=\int_{0}^{\infty} \xi_{q}(\theta) U_{A}\left(t^{q} \theta\right) d \theta, \quad \mathcal{T}_{A}(t)=q \int_{0}^{\infty} \theta \xi_{q}(\theta) U_{A}\left(t^{q} \theta\right) d \theta,
$$

$f \in \mathcal{P}_{F}(x)$, and the function $\xi_{q}$ is defined by Formula (7).

Lemma 2. (See [31], Lemma 3.4.) The operator functions $\mathcal{G}_{A}$ and $\mathcal{T}_{A}$ possess the following properties:

(1) For each $t \in[0, T]$, the operator functions $\mathcal{G}_{A}(t)$ and $\mathcal{T}_{A}(t)$ are linear bounded operators; more precisely, for each $x \in H$, we have

$$
\begin{gathered}
\left\|\mathcal{G}_{A}(t) x\right\|_{H} \leq M\|x\|_{H^{\prime}} \\
\left\|\mathcal{T}_{A}(t) x\right\|_{H} \leq \frac{q M}{\Gamma(1+q)}\|x\|_{H^{\prime}}
\end{gathered}
$$

where

$$
M=\sup _{t \in[0,+\infty)}\left\|U_{A}(t)\right\| .
$$

(2) The operator functions $\mathcal{G}_{A}(\cdot)$ and $\mathcal{T}_{A}(\cdot)$ are strongly continuous, i.e., functions $t \in[0, T] \rightarrow$ $\mathcal{G}_{A}(t) x$ and $t \in[0, T] \rightarrow \mathcal{T}_{A}(t) x$ are continuous for each $x \in H$.

Remark 2. Notice that if $A$ is a bounded operator, then the solution defined by Formula (18) satisfies the following differential equation (see [3]):

$$
{ }^{C} D_{0}^{q} x(t)=A x(t)+f(t) .
$$

Now, suppose that $x \in C([0, T] ; H)$ is any mild solution to problems (15) and (16). Take a selection $f \in \mathcal{P}_{F}(x)$ satisfying (18). Then, condition (F3) implies that

$$
\|f(t)\| \leq \omega_{R}(t) \text { for a.e. } t \in[0, T],
$$

where $R=\|x\|_{C([0, T] ; H)}$ and $\omega_{R} \in L^{\infty}(0, T)$.

Now, taking a piecewise linear function $g_{\varepsilon}$ satisfying the conditions of Lemma 3, consider the function

$$
x_{\varepsilon}(t)=\mathcal{G}_{A}(t) x_{0, \varepsilon}+\int_{0}^{t}(t-s)^{q-1} \mathcal{T}_{A}(t-s) g_{\varepsilon}(s) d s,
$$

where $x_{0, \varepsilon} \in D(A)$ and $x_{0, \varepsilon} \rightarrow x_{0}$ as $\varepsilon \rightarrow 0$.

Now, consider Yosida approximations for the operator $A$ :

$$
A_{n}=n A(n I-A)^{-1}, \quad n \geq 1 .
$$

It is known (see, e.g., $[37,38])$ that $A_{n}$ are bounded, mutually commuting operators, $A_{n}$ converges to $A$ pointwise on $D(A)$, and each $A_{n}$ generates the uniformly continuous contraction semigroup $U_{A_{n}}$.

We introduce the approximations $x_{n, \varepsilon}$ with the formula

$$
x_{n, \varepsilon}(t)=\mathcal{G}_{A_{n}}(t) x_{0, \varepsilon}+\int_{0}^{t}(t-s)^{q-1} \mathcal{T}_{A_{n}}(t-s) g_{\mathcal{\varepsilon}}(s) d s .
$$


Lemma 3. For each $\varepsilon>0$, there exists a set $m_{\varepsilon} \subset[0, T]$ of a Lebesgue measure $\mu\left(m_{\varepsilon}\right)<\varepsilon$ and a piecewise linear function $g_{\varepsilon}:[0, T] \rightarrow H$ with a finite number of nodes belonging to $D(A)$ such that

$$
\left\|f(t)-g_{\varepsilon}(t)\right\|<\varepsilon, \quad t \in[0, T] \backslash m_{\varepsilon} .
$$

Proof. Notice that we can assume, without loss of generality, that the selection $f$ is a continuous function. In fact, consider the functions $f_{\gamma}:[0, T] \rightarrow H$ defined by the formula

$$
f_{\gamma}(t)=\frac{1}{2 \gamma} \int_{t-\gamma}^{t+\gamma} \bar{f}(s) d s
$$

where

$$
\bar{f}(s)= \begin{cases}f(s), & \text { for } s \in[0, T] \\ 0, & \text { for } s \notin[0, T] .\end{cases}
$$

Recall that a point $t \in[0, T]$ is called a Lebesgue point of the function $f$ if

$$
\lim _{\gamma \rightarrow 0} \frac{1}{\gamma} \int_{t}^{t+\gamma}|f(s)-f(t)| d s=0
$$

If we rewrite

$$
f_{\gamma}(t)=\frac{1}{2 \gamma} \int_{t-\gamma}^{t+\gamma} \bar{f}(s) d s=\frac{1}{2 \gamma} \int_{t-\gamma}^{t} \bar{f}(s) d s+\frac{1}{2 \gamma} \int_{t}^{t+\gamma} \bar{f}(s) d s,
$$

then $f_{\gamma}(t) \rightarrow f(t)$ for a.e. $t \in[0, T]$ as $\gamma \rightarrow 0$, since, for a measurable function, the Lebesgue points form a complete measure space (see [39]). Notice that functions $f_{\gamma}$ are continuous and

$$
\left\|f_{\gamma}(t)\right\| \leq\|f\|_{L^{\infty}([0, T], H)} \text { for } t \in[0, T] .
$$

Hence, each function $f_{\gamma}$ may be approximated with an arbitrary degree of accuracy in the space $C([0, T] ; H)$ by piecewise linear functions $g_{\delta}$ with a finite number of nodes belonging to $D(A)$.

Take a sequence $\gamma_{k} \rightarrow 0$. Applying the Egorov theorem to functions $f_{\gamma_{k}}$ (see [37]), for a given $\varepsilon>0$, we may find $m_{\varepsilon} \subset[0, T]$ such that $\mu\left(m_{\varepsilon}\right)<\varepsilon$, and the sequence $\left\{f_{\gamma_{k}}\right\}$ uniformly converges to $f$ on $[0, T] \backslash m_{\varepsilon}$. So, we will have, for a sufficiently large $k$,

$$
\left\|f(t)-f_{\gamma_{k}}(t)\right\|<\frac{\varepsilon}{2} \text { for } t \in[0, T] \backslash m_{\varepsilon} .
$$

Taking now a piecewise linear function $g_{\varepsilon}$ satisfying

$$
\left\|f_{\gamma_{k}}-g_{\varepsilon}\right\|_{C([0, T], H)}<\frac{\varepsilon}{2}
$$

we will get the desired function.

Lemma 4. The expression

$$
I(\varepsilon)(t):=\int_{0}^{t}(t-s)^{q-1}\left\|\mathcal{T}_{A}(t-s)\right\| \cdot\left\|g_{\varepsilon}(s)-f(s)\right\| d s
$$

tends to zero as $\varepsilon \rightarrow 0$ uniformly on $[0, T]$.

Proof. Denoting

$$
N=\frac{q M}{\Gamma(1+q)}
$$

we get from (20) that

$$
\left\|\mathcal{T}_{A}(t)\right\| \leq N, \quad t \in[0, T]
$$


For a given $\gamma>0$, we choose $\sigma>0$ such that

$$
\frac{\sigma^{q}}{q} N\left(2\left\|\omega_{R}\right\|_{L^{\infty}}+1\right)<\frac{\gamma}{2}
$$

From the construction of the function $g_{\varepsilon}$ (see relations (26) and (27)), it follows that for a sufficiently small $\varepsilon>0$, we get

$$
\left\|g_{\mathcal{E}}(t)\right\| \leq\left\|\omega_{R}\right\|_{L^{\infty}}+1, \quad t \in[0, T] .
$$

Then, for the case $t \leq \sigma \leq T$, we have

$$
\begin{gathered}
\int_{0}^{t}(t-s)^{q-1}\left\|\mathcal{T}_{A}(t-s)\right\| \cdot\left\|g_{\mathcal{\varepsilon}}(s)-f(s)\right\| d s \leq \int_{0}^{\sigma}(t-s)^{q-1} N\left(\left\|g_{\mathcal{\varepsilon}}(s)\right\|+\|f(s)\|\right) d s \\
\leq N\left(2\left\|\omega_{R}\right\|_{L^{\infty}}+1\right) \int_{0}^{\sigma}(t-s)^{q-1} d s=\frac{\sigma^{q}}{q} N\left(2\left\|\omega_{R}\right\|_{L^{\infty}}+1\right)<\frac{\gamma}{2} .
\end{gathered}
$$

If $\sigma<t$, we get

$$
\begin{gathered}
I(\varepsilon)(t)=\int_{t-\sigma}^{t}(t-s)^{q-1}\left\|\mathcal{T}_{A}(t-s)\right\| \cdot\left\|g_{\varepsilon}(s)-f(s)\right\| d s \\
+\int_{0}^{t-\sigma}(t-s)^{q-1}\left\|\mathcal{T}_{A}(t-s)\right\| \cdot\left\|g_{\varepsilon}(s)-f(s)\right\| d s=I_{1}(\varepsilon)(t)+I_{2}(\varepsilon)(t) .
\end{gathered}
$$

For $I_{1}(\varepsilon)$, the following estimate holds:

$$
I_{1}(\varepsilon)(t) \leq \frac{\sigma^{q}}{q} N\left(2\left\|\omega_{R}\right\|_{L^{\infty}}+1\right)<\frac{\gamma}{2} .
$$

For $I_{2}(\varepsilon)$, we have

$$
\begin{gathered}
I_{2}(\varepsilon)(t)=\int_{[0, t-\sigma] \backslash m_{\varepsilon}}(t-s)^{q-1}\left\|\mathcal{T}_{A}(t-s)\right\| \cdot\left\|g_{\varepsilon}(s)-f(s)\right\| d s \\
+\int_{[0, t-\sigma] \cap m_{\varepsilon}}(t-s)^{q-1}\left\|\mathcal{T}_{A}(t-s)\right\| \cdot\left\|g_{\varepsilon}(s)-f(s)\right\| d s=I_{21}(\varepsilon)(t)+I_{22}(\varepsilon)(t) .
\end{gathered}
$$

Set

$$
N_{1}=\max _{s \in[0, t-\sigma]}(t-s)^{q-1}\left\|\mathcal{T}_{A}(t-s)\right\| .
$$

Since $\left\|g_{\mathcal{\varepsilon}}(s)-f(s)\right\|<\varepsilon$ for $s \in[0, t-\sigma] \backslash m_{\varepsilon}$, we obtain the estimate

$$
I_{21}(\varepsilon)(t) \leq N_{1} \varepsilon(t-\sigma)<N_{1} \varepsilon T .
$$

For $I_{22}(\varepsilon)$, we have

$$
I_{22}(\varepsilon)(t) \leq N_{1}\left(2\left\|\omega_{R}\right\|_{L^{\infty}}+1\right) \mu\left([0, t-\sigma] \cap m_{\varepsilon}\right) \leq N_{1}\left(2\left\|\omega_{R}\right\|_{L^{\infty}}+1\right) \varepsilon
$$

Now, choosing $\varepsilon$ so that

$$
N_{1}\left(T+2\left\|\omega_{R}\right\|_{L^{\infty}}+1\right) \varepsilon<\frac{\gamma}{2}
$$

we get the desired statement.

Corollary 1. The expression $\left\|x_{\varepsilon}-x\right\|_{C([0, T] ; H)}$ tends to zero as $\varepsilon \rightarrow 0$. 
Proof. We have the estimate

$$
\left\|x(t)-x_{\varepsilon}(t)\right\| \leq\left\|\mathcal{G}_{A}(t)\left(x_{0}-x_{0, \varepsilon}\right)\right\|+\int_{0}^{t}(t-s)^{q-1}\left\|\mathcal{T}_{A}(t-s)\right\| \cdot\left\|g_{\mathcal{\varepsilon}}(s)-f(s)\right\| d s .
$$

Since the operator function $\mathcal{G}_{A}(t)$ is strongly continuous and $x_{0, \varepsilon} \rightarrow x_{0}$ as $\varepsilon \rightarrow 0$, the first term in this sum tends to zero uniformly on $[0, T]$. The second term uniformly tends to zero due to Lemma 4.

Remark 3. If in Lemma 4, we replace $\left\|\mathcal{T}_{A}(t-s)\right\|$ with the Mittag-Leffler function $E_{q, q}((-d+$ a) $\left.t^{q}\right)$, then, repeating the above reasonings, we get

$$
\int_{0}^{t}(t-s)^{q-1} E_{q, q}\left((-d+a)(t-s)^{q}\right) \cdot\left\|g_{\varepsilon}(s)-f(s)\right\| d s \rightarrow 0
$$

as $\varepsilon \rightarrow 0$ uniformly on $[0, T]$.

Lemma 5. For a fixed $\varepsilon>0$, the sequence $x_{n, \varepsilon}$ converges to $x_{\varepsilon}$ as $n \rightarrow \infty$ uniformly on $[0, T]$.

Proof. Since for each fixed $x \in H$, we have

$$
U_{A_{n}}(t) x \rightarrow U_{A}(t) x
$$

uniformly with respect to $t \in[0, T]$ (see [38]), we also have the uniform convergence

$$
\begin{aligned}
\mathcal{G}_{A_{n}}(t) x & \rightarrow \mathcal{G}_{A}(t) x, \\
\mathcal{T}_{A_{n}}(t) x & \rightarrow \mathcal{T}_{A}(t) x,
\end{aligned}
$$

which implies the desired convergence for a fixed $\varepsilon>0$.

Notice that due to the closedness of the operator $A$, we have for $x \in D(A)$ :

$$
\begin{aligned}
A U_{A}(t) x & =U_{A}(t) A x, \\
A A_{n} x & =A_{n} A x, \\
A U_{A_{n}}(t) x & =U_{A_{n}}(t) A x .
\end{aligned}
$$

By the definition of the operator functions $\mathcal{G}_{A}(t)$ and $\mathcal{T}_{A}(t)$, we have for $x \in D(A)$ :

$$
\begin{aligned}
A \mathcal{G}_{A}(t) x & =\mathcal{G}_{A}(t) A x ; \\
A \mathcal{T}_{A}(t) x & =\mathcal{T}_{A}(t) A x ; \\
A \mathcal{G}_{A_{n}}(t) x & =\mathcal{G}_{A_{n}}(t) A x ; \\
A \mathcal{T}_{A_{n}}(t) x & =\mathcal{T}_{A_{n}}(t) A x
\end{aligned}
$$

Since for a given piecewise linear function $g_{\varepsilon}$, the set $\left\{A g_{\varepsilon}(s): s \in[0, T]\right\}$ is compact in $H$, it follows that the range of the function $g_{\varepsilon}$ lies in $D(A)$, and therefore, $\left\{A x_{n, \varepsilon}(t): t \in[0, T]\right\}$ is a compact set.

Lemma 6. For a fixed $\varepsilon>0$, we have

$$
\left(n(n I-A)^{-1}-I\right) A x_{n, \varepsilon}(t) \rightarrow 0
$$

as $n \rightarrow \infty$ uniformly with respect to $t \in[0, T]$. 
Proof. Since $\left(n(n I-A)^{-1}-I\right) y \rightarrow 0$ for each fixed $y \in H$ (see [38]), we have

$$
\left(n(n I-A)^{-1}-I\right) A x \rightarrow 0
$$

uniformly with respect to $x \in\left\{g_{\varepsilon}(t): t \in[0, T]\right\}$. Since $x_{n, \varepsilon}$ can be expressed through $g_{\varepsilon}$ by the Formula (24), we get thedesired convergence.

Now, we can get the following assertion about the a priori estimate of a solution to problems (15) and (16).

Theorem 1. Under the above conditions, there exists a continuous function $\mathcal{C}:[0,+\infty) \rightarrow$ $[0,+\infty)$ such that

$$
\|x\|_{C([0, T], H)} \leq \mathcal{C}(T) .
$$

for every mild solution $x$ of problems (15) and (16) defined on an interval $[0, T]$.

Proof. Take a sequence of positive numbers $\theta_{k} \rightarrow 0$ and choose a sequence of approximations $\left\{x_{\varepsilon_{k}}\right\}$ so that

$$
\left\|x_{\varepsilon_{k}}-x\right\|_{C([0, T], H)}<\theta_{k} .
$$

Further, according to Lemma 4 , we find $n^{1}\left(\varepsilon_{k}\right)$ such that for $n \geq n^{1}\left(\varepsilon_{k}\right)$, the following holds:

$$
\left\|x_{n, \varepsilon_{k}}-x_{\varepsilon_{k}}\right\|_{C([0, T], H)}<\theta_{k} .
$$

Since $x_{n, \varepsilon}$ lie in $D(A)$ and $A x_{n, \varepsilon}$, for each fixed $\varepsilon$ that is uniformly bounded in $n$ (see Lemma 6), we may indicate $n^{2}\left(\varepsilon_{k}\right)$ such that for $n \geq n^{2}\left(\varepsilon_{k}\right)$, we have

$$
\sup _{t \in[0, T]}\left\langle\left(n(n I-A)^{-1}-I\right) A x_{n, \varepsilon}(t), x_{n, \varepsilon}(t)\right\rangle<\theta_{k} .
$$

Take $n_{k}=\max \left(n^{1}\left(\varepsilon_{k}\right), n^{2}\left(\varepsilon_{k}\right)\right)$.

Then, we get

$$
\begin{gathered}
\left\|x_{n_{k}, \varepsilon_{k}}-x_{\varepsilon_{k}}\right\|_{C([0, T], H)}<\theta_{k}, \\
\sup _{t \in[0, T]}\left\langle\left(n_{k}\left(n_{k} I+A\right)^{-1}-I\right) A x_{n_{k}, \varepsilon_{k}}(t), x_{n_{k}, \varepsilon_{k}}(t)\right\rangle<\theta_{k} .
\end{gathered}
$$

Notice that, simultaneously, we construct the corresponding sequences of functions $\left\{g_{\varepsilon_{k}}\right\}$ and sets $m_{\varepsilon_{k}}$.

By virtue of Remark 2, we have

$$
{ }^{C} D_{0}^{q} x_{n_{k}, \varepsilon_{k}}(t)=A_{n_{k}} x_{n_{k}, \varepsilon_{k}}(t)+g_{\varepsilon_{k}}(t) .
$$

From $[25,26]$, it follows that

$$
{ }^{C} D_{0}^{q}\left\|x_{n_{k}, \varepsilon_{k}}(t)\right\|^{2} \leq\left\langle A_{n_{k}} x_{n_{k}, \varepsilon_{k}}(t), x_{n_{k}, \varepsilon_{k}}(t)\right\rangle+\left\langle g_{\varepsilon_{k}}(t), x_{n_{k}, \varepsilon_{k}}(t)\right\rangle .
$$

Now, let us estimate the right-hand side of inequality (31). To do this, let us mention that condition F5) implies that

$\left.F 5^{\prime}\right)$ for each $x \in H$ and $y \in F(t, x), t \in[0, T]$ it holds

$$
\langle y, x\rangle \leq a\|x\|^{2}+G(t),
$$

where $G(t)=\|F(t, 0)\|$.

Then, we get

$$
\left\langle A_{n_{k}} x_{n_{k}, \varepsilon_{k}}(t), x_{n_{k}, \varepsilon_{k}}(t)\right\rangle+\left\langle g_{\varepsilon_{k}}(t), x_{n_{k}, \varepsilon_{k}}(t)\right\rangle=\left\langle A x_{n_{k}, \varepsilon_{k}}(t), x_{n_{k}, \varepsilon_{k}}(t)\right\rangle
$$




$$
\begin{gathered}
+\left\langle\left(n_{k}\left(n_{k} I-A\right)^{-1}-I\right) A x_{n_{k}, \varepsilon_{k}}(t), x_{n_{k}, \varepsilon_{k}}(t)\right\rangle \\
+\left\langle g_{\varepsilon_{k}}(t)-f(t), x_{n_{k}, \varepsilon_{k}}(t)\right\rangle+\langle f(t), x(t)\rangle+\left\langle f(t), x_{n_{k}, \varepsilon_{k}}(t)-x(t)\right\rangle \\
\leq-d\left\|x_{n_{k}, \varepsilon_{k}}(t)\right\|^{2}+a\|x(t)\|^{2}+\left\langle g_{\varepsilon_{k}}(t)-f(t), x_{n_{k}, \varepsilon_{k}}(t)\right\rangle+\left\langle f(t), x_{n_{k}, \varepsilon_{k}}(t)-x(t)\right\rangle \\
+\left\langle\left(n_{k}\left(n_{k} I-A\right)^{-1}-I\right) A x_{n_{k}, \varepsilon_{k}}(t), x_{n_{k}, \varepsilon_{k}}(t)\right\rangle+G(t) \\
\leq(-d+a)\left\|x_{n_{k}, \varepsilon_{k}}(t)\right\|^{2}+a\left\|x(t)-x_{n_{k}, \varepsilon_{k}}(t)\right\|\left(\|x(t)\|+\left\|x_{n_{k}, \varepsilon_{k}}(t)\right\|\right)+\left\|g_{\varepsilon_{k}}(t)-f(t)\right\|\left\|x_{n_{k}, \varepsilon_{k}}(t)\right\| \\
+\|f(t)\|\left\|x_{n_{k}, \varepsilon_{k}}(t)-x(t)\right\|+\left\langle\left(n_{k}\left(n_{k} I-A\right)^{-1}-I\right) A x_{n_{k}, \varepsilon_{k}}(t), x_{n_{k}, \varepsilon_{k}}(t)\right\rangle+G(t) .
\end{gathered}
$$

For sufficiently large $k$, we get the inequality

$$
\begin{aligned}
{ }^{C} D_{0}^{q}\left\|x_{n_{k}, \varepsilon_{k}}(t)\right\|^{2} \leq & (-d+a)\left\|x_{n_{k}, \varepsilon_{k}}(t)\right\|^{2}+3 a R \theta_{k}+\theta_{k}\|f(t)\|+\theta_{k} \\
& +2 R\left\|g_{\varepsilon_{k}}(t)-f(t)\right\|+G(t) .
\end{aligned}
$$

By virtue of Lemma 1, assuming that

$$
\begin{gathered}
z(t)=\left\|x_{n_{k}, \varepsilon_{k}}(t)\right\|^{2}, \lambda=-d+a, \\
l(t)=3 a R \theta_{k}+\theta_{k}\|f(t)\|+\theta_{k}+2 R\left\|g_{\varepsilon_{k}}(t)-f(t)\right\|+G(t),
\end{gathered}
$$

the following inequality holds true:

$$
\begin{aligned}
\left\|x_{n_{k}, \varepsilon_{k}}(t)\right\|^{2} \leq & E_{q}\left((-d+a) t^{q}\right)\left\|x_{0, \varepsilon_{k}}\right\|^{2}+\int_{0}^{t}(t-s)^{q-1} E_{q, q}\left((-d+a)(t-s)^{q}\right) G(s) d s+ \\
+ & \theta_{k} \int_{0}^{t}(t-s)^{q-1} E_{q, q}\left((-d+a)(t-s)^{q}\right)\left(3 a R+\omega_{R}(s)+1\right) d s+ \\
& +2 R \int_{0}^{t}(t-s)^{q-1} E_{q, q}\left((-d+a)(t-s)^{q}\right)\left\|g_{\varepsilon_{k}}(s)-f(s)\right\| d s
\end{aligned}
$$

Notice that the third and fourth terms tend to zero as $k \rightarrow \infty$. In fact, in the third term, the integral is uniformly bounded on $[0, T]$ and we can apply Remark 3 to the fourth term.

Passing in (39) to the limit as $k \rightarrow \infty$, we get

$$
\|x(t)\|^{2} \leq E_{q}\left((-d+a) t^{q}\right)\left\|x_{0}\right\|+\int_{0}^{t}(t-s)^{q-1} E_{q, q}\left((-d+a)(t-s)^{q}\right) G(s) d s .
$$

Therefore, the right-hand side determines the function of the a priori estimate $\mathcal{C}$ on the interval $[0, T]$.

From Theorem 1, we can obtain the following result on the existence of a solution to problems (15) and (16) on an arbitrary interval $[0, T]$.

Theorem 2. Under the above conditions, there exists a mild solution to problems (15) and (16) on $[0, T]$ for each $T>0$.

Proof. Consider the family of multivalued integral operators $\mathcal{F}: C([0, T] ; H) \times[0,1] \rightarrow P(C([0, T] ; H))$ defined in the following way:

$$
\mathcal{F}(x, \lambda)=\left\{z=\mathcal{G}_{A}(t) x_{0}+\lambda \int_{0}^{t}(t-s)^{q-1} \mathcal{T}_{A}(t-s) f(s) d s: f \in \mathcal{P}_{F}(x)\right\},
$$

where $\mathcal{P}_{F}$ is the superposition multioperator defined by (17).

It is clear that each fixed point $x_{\lambda} \in C([0, T] ; H)$ of the multimap $\mathcal{F}(\cdot, \lambda), \lambda \in[0,1]$ is a mild solution to the problem

$$
{ }^{C} D_{0}^{q} x(t) \in A x(t)+\lambda F(t, x(t)), \quad t \in[0, T]
$$




$$
x(0)=x_{0} .
$$

Moreover, it is known (see [5,8,10-14]) that the family (34) has compact convex values and is condensing with respect to the MNC $v$ in $C([0, T] ; H)$ (see Section 2). Since the multioperators $\lambda F$ satisfy conditions $(F 1)-(F 5)$ independently on $\lambda$, by applying Theorem 1 , we conclude that there exists a constant $\mathcal{C}(T)$ such that all solutions to problems (35) and (36) satisfy the a priori estimate

$$
\left\|x_{\lambda}\right\| \leq \mathcal{C}(T) .
$$

So, the multioperators $\mathcal{F}(\cdot, \lambda)$ from family (34) are fixed-point free on the boundary of the ball $\mathcal{B}$ of the space $C([0, T] ; H)$ centered at zero of the radius $\mathcal{C}(T)+1$. Notice that the range of the multioperator $\mathcal{F}(\cdot, 0)$ consists of the single function $y(t)=\mathcal{G}_{A}(t) x_{0}$ as its fixed point.

Now, applying the homotopy and normalization properties of the topological degree, we obtain

$$
\operatorname{deg}(i-\mathcal{F}(\cdot, 1), \mathcal{B})=\operatorname{deg}(i-\mathcal{F}(\cdot, 0), \mathcal{B})=1,
$$

which yields, by the existence property of the topological degree, the desired result.

\section{Uniqueness of a Solution}

Now, we are in position to present our main result.

Theorem 3. Under the above conditions, problems (15) and (16) have a unique mild solution on $[0, T]$ for each $T>0$.

Proof. Suppose the contrary, that there are two different mild solutions $x^{1}, x^{2}$ on $[0, T]$ for problems (15) and (16). Take a sequence of positive numbers $\theta_{k} \rightarrow 0$ and choose a sequences of approximations $\left\{x_{\varepsilon_{k}}^{1}\right\}$ and $\left\{x_{\varepsilon_{k}}^{2}\right\}$ so that

$$
\left\|x_{\varepsilon_{k}}^{1}-x^{1}\right\|_{C([0, T], H)}<\theta_{k},\left\|x_{\varepsilon_{k}}^{2}-x^{2}\right\|_{C([0, T], H)}<\theta_{k} .
$$

Further, according to Lemma 4 , we find $n^{1}\left(\varepsilon_{k}\right)$ such that for $n \geq n^{1}\left(\varepsilon_{k}\right)$, the following holds:

$$
\left\|x_{n, \varepsilon_{k}}^{1}-x_{\varepsilon_{k}}^{1}\right\|_{C([0, T], H)}<\theta_{k},\left\|x_{n, \varepsilon_{k}}^{2}-x_{\varepsilon_{k}}^{2}\right\|_{C([0, T], H)}<\theta_{k} .
$$

Since $x_{n, \varepsilon}^{1}, x_{n, \varepsilon}^{1} \in D(A)$ and $A x_{n, \varepsilon}^{1}, A x_{n, \varepsilon}^{2}$ for each fixed $\varepsilon$ that is uniformly bounded in $n$ (see Lemma 6), we may indicate $n^{2}\left(\varepsilon_{k}\right)$ such that for $n \geq n^{2}\left(\varepsilon_{k}\right)$, we have

$$
\begin{aligned}
& \sup _{t \in[0, T]}\left\langle\left(n(n I-A)^{-1}-I\right) A x_{n, \varepsilon}^{1}(t), x_{n, \varepsilon}^{1}(t)\right\rangle<\theta_{k}, \\
& \sup _{t \in[0, T]}\left\langle\left(n(n I-A)^{-1}-I\right) A x_{n, \varepsilon}^{2}(t), x_{n, \varepsilon}^{2}(t)\right\rangle<\theta_{k} .
\end{aligned}
$$

Take $n_{k}=\max \left(n^{1}\left(\varepsilon_{k}\right), n^{2}\left(\varepsilon_{k}\right)\right)$.

Then, we get

$$
\begin{gathered}
\left\|x_{n_{k}, \varepsilon_{k}}^{1}-x_{\varepsilon_{k}}^{1}\right\|_{C([0, T], H)}<\theta_{k},\left\|x_{n_{k}, \varepsilon_{k}}^{2}-x_{\varepsilon_{k}}^{2}\right\|_{C([0, T], H)}<\theta_{k}, \\
\sup _{t \in[0, T]}\left\langle\left(n_{k}\left(n_{k} I+A\right)^{-1}-I\right) A x_{n_{k}, \varepsilon_{k}}^{1}(t), x_{n_{k}, \varepsilon_{k}}^{1}(t)\right\rangle<\theta_{k}, \\
\sup _{t \in[0, T]}\left\langle\left(n_{k}\left(n_{k} I+A\right)^{-1}-I\right) A x_{n_{k}, \varepsilon_{k}}^{2}(t), x_{n_{k}, \varepsilon_{k}}^{2}(t)\right\rangle<\theta_{k} .
\end{gathered}
$$

Notice that, simultaneously, we construct the corresponding sequences of functions $\left\{g_{\varepsilon_{k}}^{1}\right\},\left\{g_{\varepsilon_{k}}^{2}\right\}$ and sets $m_{\varepsilon_{k}}$. 
By virtue of Remark 2, we have

$$
\begin{aligned}
& { }^{C} D_{0}^{q} x_{n_{k}, \varepsilon_{k}}^{1}(t)=A_{n_{k}} x_{n_{k}, \varepsilon_{k}}^{1}(t)+g_{\varepsilon_{k}}^{1}(t), \\
& { }^{C} D_{0}^{q} x_{n_{k}, \varepsilon_{k}}^{2}(t)=A_{n_{k}} x_{n_{k}, \varepsilon_{k}}^{2}(t)+g_{\varepsilon_{k}}^{2}(t) .
\end{aligned}
$$

From $[25,26]$, it follows that

$$
\begin{gathered}
{ }^{C} D_{0}^{q}\left\|x_{n_{k}, \varepsilon_{k}}^{1}(t)-x_{n_{k}, \varepsilon_{k}}^{2}(t)\right\|^{2} \leq\left\langle A_{n_{k}}\left(x_{n_{k}, \varepsilon_{k}}^{1}(t)-x_{n_{k}, \varepsilon_{k}}^{2}(t)\right), x_{n_{k}, \varepsilon_{k}}^{1}(t)-x_{n_{k}, \varepsilon_{k}}^{2}(t)\right\rangle \\
+\left\langle g_{\varepsilon_{k}}^{1}(t)-g_{\varepsilon_{k}}^{2}(t), x_{n_{k}, \varepsilon_{k}}^{1}(t)-x_{n_{k}, \varepsilon_{k}}^{2}(t)\right\rangle .
\end{gathered}
$$

Now, let us estimate the right-hand side of inequality (38). Using the properties of Yosida approximation for the first term and adding and subtracting $f^{1}(t)-f^{2}(t)$ in the second term, we have

$$
\begin{gathered}
\left\langle A_{n_{k}}\left(x_{n_{k}, \varepsilon_{k}}^{1}(t)-x_{n_{k}, \varepsilon_{k}}^{2}(t)\right), x_{n_{k}, \varepsilon_{k}}^{1}(t)-x_{n_{k}, \varepsilon_{k}}^{2}(t)\right\rangle+\left\langle g_{\varepsilon_{k}}^{1}(t)-g_{\varepsilon_{k}}^{2}(t), x_{n_{k}, \varepsilon_{k}}^{1}(t)-x_{n_{k}, \varepsilon_{k}}^{2}(t)\right\rangle \\
=\left\langle A\left(x_{n_{k}, \varepsilon_{k}}^{1}(t)-x_{n_{k}, \varepsilon_{k}}^{2}(t)\right), x_{n_{k}, \varepsilon_{k}}^{1}(t)-x_{n_{k}, \varepsilon_{k}}^{2}(t)\right\rangle \\
+\left\langle\left(n_{k}\left(n_{k} I-A\right)^{-1}-I\right) A\left(x_{n_{k}, \varepsilon_{k}}^{1}(t)-x_{n_{k}, \varepsilon_{k}}^{2}(t)\right), x_{n_{k}, \varepsilon_{k}}^{1}(t)-x_{n_{k}, \varepsilon_{k}}^{2}(t)\right\rangle \\
+\left\langle g_{\varepsilon_{k}}^{1}(t)-g_{\varepsilon_{k}}^{2}(t)-\left(f^{1}(t)-f^{2}(t)\right)+\left(f^{1}(t)-f^{2}(t)\right), x_{n_{k}, \varepsilon_{k}}^{1}(t)-x_{n_{k}, \varepsilon_{k}}^{2}(t)\right\rangle \\
=\left\langle A\left(x_{n_{k}, \varepsilon_{k}}^{1}(t)-x_{n_{k}, \varepsilon_{k}}^{2}(t)\right), x_{n_{k}, \varepsilon_{k}}^{1}(t)-x_{n_{k}, \varepsilon_{k}}^{2}(t)\right\rangle \\
+\left\langle\left(n_{k}\left(n_{k} I-A\right)^{-1}-I\right) A x_{n_{k}, \varepsilon_{k}}^{1}(t), x_{n_{k}, \varepsilon_{k}}^{1}(t)-x_{n_{k}, \varepsilon_{k}}^{2}(t)\right\rangle \\
-\left\langle\left(n_{k}\left(n_{k} I-A\right)^{-1}-I\right) A x_{n_{k}, \varepsilon_{k}}^{2}(t), x_{n_{k}, \varepsilon_{k}}^{1}(t)-x_{n_{k}, \varepsilon_{k}}^{2}(t)\right\rangle \\
+\left\langle g_{\varepsilon_{k}}^{1}(t)-f^{1}(t), x_{n_{k}, \varepsilon_{k}}^{1}(t)-x_{n_{k}, \varepsilon_{k}}^{2}(t)\right\rangle \\
+\left\langle f^{2}(t)-g_{\varepsilon_{k}}^{2}(t), x_{n_{k}, \varepsilon_{k}}^{1}(t)-x_{n_{k}, \varepsilon_{k}}^{2}(t)\right\rangle \\
+\left\langle f^{1}(t)-f^{2}(t), x_{n_{k}, \varepsilon_{k}}^{1}(t)-x_{n_{k}, \varepsilon_{k}}^{2}(t)\right\rangle .
\end{gathered}
$$

Adding and subtracting $x^{1}(t)-x^{2}(t)$ in the last term, we get

$$
\begin{gathered}
\left\langle A_{n_{k}}\left(x_{n_{k}, \varepsilon_{k}}^{1}(t)-x_{n_{k}, \varepsilon_{k}}^{2}(t)\right), x_{n_{k}, \varepsilon_{k}}^{1}(t)-x_{n_{k}, \varepsilon_{k}}^{2}(t)\right\rangle+\left\langle g_{\varepsilon_{k}}^{1}(t)-g_{\varepsilon_{k}}^{2}(t), x_{n_{k}, \varepsilon_{k}}^{1}(t)-x_{n_{k}, \varepsilon_{k}}^{2}(t)\right\rangle \\
=\left\langle A\left(x_{n_{k}, \varepsilon_{k}}^{1}(t)-x_{n_{k}, \varepsilon_{k}}^{2}(t)\right), x_{n_{k}, \varepsilon_{k}}^{1}(t)-x_{n_{k}, \varepsilon_{k}}^{2}(t)\right\rangle \\
+\left\langle\left(n_{k}\left(n_{k} I-A\right)^{-1}-I\right) A x_{n_{k}, \varepsilon_{k}}^{1}(t), x_{n_{k}, \varepsilon_{k}}^{1}(t)-x_{n_{k}, \varepsilon_{k}}^{2}(t)\right\rangle \\
-\left\langle\left(n_{k}\left(n_{k} I-A\right)^{-1}-I\right) A x_{n_{k}, \varepsilon_{k}}^{2}(t), x_{n_{k}, \varepsilon_{k}}^{1}(t)-x_{n_{k}, \varepsilon_{k}}^{2}(t)\right\rangle \\
+\left\langle g_{\varepsilon_{k}}^{1}(t)-f^{1}(t), x_{n_{k}, \varepsilon_{k}}^{1}(t)-x_{n_{k}, \varepsilon_{k}}^{2}(t)\right\rangle \\
\quad+\left\langle f^{2}(t)-g_{\varepsilon_{k}}^{2}(t), x_{n_{k}, \varepsilon_{k}}^{1}(t)-x_{n_{k}, \varepsilon_{k}}^{2}(t)\right\rangle \\
+\left\langle f^{1}(t)-f^{2}(t), x_{n_{k}, \varepsilon_{k}}^{1}(t)-x_{n_{k}, \varepsilon_{k}}^{2}(t)-\left(x^{1}(t)-x^{2}(t)\right)\right\rangle+\left\langle f^{1}(t)-f^{2}(t), x^{1}(t)-x^{2}(t)\right\rangle .
\end{gathered}
$$

Using the properties $(A)$ and $(F 5)$, we have

$$
\begin{aligned}
\left\langle A_{n_{k}}\left(x_{n_{k}, \varepsilon_{k}}^{1}(t)-x_{n_{k}, \varepsilon_{k}}^{2}(t)\right), x_{n_{k}, \varepsilon_{k}}^{1}(t)-x_{n_{k}, \varepsilon_{k}}^{2}(t)\right\rangle+\left\langle g_{\varepsilon_{k}}^{1}(t)-g_{\varepsilon_{k}}^{2}(t), x_{n_{k}, \varepsilon_{k}}^{1}(t)-x_{n_{k}, \varepsilon_{k}}^{2}(t)\right\rangle \\
\leq-d\left\|x_{n_{k}, \varepsilon_{k}}^{1}(t)-x_{n_{k}, \varepsilon_{k}}^{2}(t)\right\|^{2}+a\left\|x^{1}(t)-x^{2}(t)\right\|^{2} \\
+\left\langle g_{\varepsilon_{k}}^{1}(t)-f^{1}(t), x_{n_{k}, \varepsilon_{k}}^{1}(t)-x_{n_{k}, \varepsilon_{k}}^{2}(t)\right\rangle \\
+\left\langle f^{2}(t)-g_{\varepsilon_{k}}^{2}(t), x_{n_{k}, \varepsilon_{k}}^{1}(t)-x_{n_{k}, \varepsilon_{k}}^{2}(t)\right\rangle
\end{aligned}
$$




$$
\begin{aligned}
+\left\langle f^{1}(t)\right. & \left.-f^{2}(t), x_{n_{k}, \varepsilon_{k}}^{1}(t)-x^{1}(t)\right\rangle+\left\langle f^{1}(t)-f^{2}(t), x^{2}(t)-x_{n_{k}, \varepsilon_{k}}^{2}(t)\right\rangle \\
+ & \left\langle\left(n_{k}\left(n_{k} I-A\right)^{-1}-I\right) A x_{n_{k}, \varepsilon_{k}}^{1}(t), x_{n_{k}, \varepsilon_{k}}^{1}(t)-x_{n_{k}, \varepsilon_{k}}^{2}(t)\right\rangle \\
& -\left\langle\left(n_{k}\left(n_{k} I-A\right)^{-1}-I\right) A x_{n_{k}, \varepsilon_{k}}^{2}(t), x_{n_{k}, \varepsilon_{k}}^{1}(t)-x_{n_{k}, \varepsilon_{k}}^{2}(t)\right\rangle .
\end{aligned}
$$

Now, using the properties of the norm and the scalar product, we finally obtain

$$
\begin{gathered}
\left\langle A_{n_{k}}\left(x_{n_{k}, \varepsilon_{k}}^{1}(t)-x_{n_{k}, \varepsilon_{k}}^{2}(t)\right), x_{n_{k}, \varepsilon_{k}}^{1}(t)-x_{n_{k}, \varepsilon_{k}}^{2}(t)\right\rangle+\left\langle g_{\varepsilon_{k}}^{1}(t)-g_{\varepsilon_{k}}^{2}(t), x_{n_{k}, \varepsilon_{k}}^{1}(t)-x_{n_{k}, \varepsilon_{k}}^{2}(t)\right\rangle \\
\leq(-d+a)\left\|x_{n_{k}, \varepsilon_{k}}^{1}(t)-x_{n_{k}, \varepsilon_{k}}^{2}(t)\right\|^{2} \\
+a\left(\left\|x^{1}(t)-x_{n_{k}, \varepsilon_{k}}^{1}(t)\right\|+\left\|x^{2}(t)-x_{n_{k}, \varepsilon_{k}}^{2}(t)\right\|\right)\left(\left\|x^{1}(t)-x^{2}(t)\right\|+\left\|x_{n_{k}, \varepsilon_{k}}^{1}(t)-x_{n_{k}, \varepsilon_{k}}^{2}(t)\right\|\right) \\
+\left\|g_{\varepsilon_{k}}^{1}(t)-f^{1}(t)\right\|\left\|x_{n_{k}, \varepsilon_{k}}^{1}(t)-x_{n_{k}, \varepsilon_{k}}^{2}(t)\right\|+\left\|f^{2}(t)-g_{\varepsilon_{k}}^{2}(t)\right\|\left\|x_{n_{k}, \varepsilon_{k}}^{1}(t)-x_{n_{k}, \varepsilon_{k}}^{2}(t)\right\| \\
+\left\|f^{1}(t)-f^{2}(t)\right\|\left\|x_{n_{k}, \varepsilon_{k}}^{1}(t)-x^{1}(t)\right\|+\left\|f^{1}(t)-f^{2}(t)\right\|\left\|x^{2}(t)-x_{n_{k}, \varepsilon_{k}}^{2}(t)\right\| \\
+\left\langle\left(n_{k}\left(n_{k} I-A\right)^{-1}-I\right) A x_{n_{k}, \varepsilon_{k}}^{1}(t), x_{n_{k}, \varepsilon_{k}}^{1}(t)-x_{n_{k}, \varepsilon_{k}}^{2}(t)\right\rangle \\
+\left\langle\left(n_{k}\left(n_{k} I-A\right)^{-1}-I\right) A x_{n_{k}, \varepsilon_{k}}^{2}(t), x_{n_{k}, \varepsilon_{k}}^{1}(t)-x_{n_{k}, \varepsilon_{k}}^{2}(t)\right\rangle .
\end{gathered}
$$

For sufficiently large $k$, we get the inequality

$$
\begin{gathered}
{ }^{C} D_{0}^{q}\left\|x_{n_{k}, \varepsilon_{k}}^{1}(t)-x_{n_{k}, \varepsilon_{k}}^{2}(t)\right\|^{2} \leq(-d+a)\left\|x_{n_{k}, \varepsilon_{k}}^{1}(t)-x_{n_{k}, \varepsilon_{k}}^{2}(t)\right\|^{2}+6 a R \theta_{k}+6 a R \theta_{k} \\
+4 R\left\|g_{\varepsilon_{k}}^{1}(t)-f^{1}(t)\right\|+4 R\left\|f^{2}(t)-g_{\varepsilon_{k}}^{2}(t)\right\|+\theta_{k}\left\|f^{1}(t)-f^{2}(t)\right\|+\theta_{k}\left\|f^{1}(t)-f^{2}(t)\right\|+2 \theta_{k} \\
=(-d+a)\left\|x_{n_{k}, \varepsilon_{k}}^{1}(t)-x_{n_{k}, \varepsilon_{k}}^{2}(t)\right\|^{2}+12 a R \theta_{k}+4 R\left\|g_{\varepsilon_{k}}^{1}(t)-f^{1}(t)\right\|+4 R\left\|f^{2}(t)-g_{\varepsilon_{k}}^{2}(t)\right\| \\
+2 \theta_{k}\left\|f^{1}(t)-f^{2}(t)\right\|+2 \theta_{k} .
\end{gathered}
$$

By virtue of the analog of Lemma 1, the following inequality holds true:

$$
\begin{gathered}
\left\|x_{n_{k}, \varepsilon_{k}}^{1}(t)-x_{n_{k}, \varepsilon_{k}}^{2}(t)\right\|^{2} \leq E_{q}\left((-d+a) t^{q}\right)\left\|x_{0, \varepsilon_{k}}-x_{0, \varepsilon_{k}}\right\|^{2} \\
+4 R \int_{0}^{t}(t-s)^{q-1} E_{q, q}\left((-d+a)(t-s)^{q}\right)\left\|g_{\varepsilon_{k}}^{1}(t)-f^{1}(t)\right\| d s+ \\
+\theta_{k} \int_{0}^{t}(t-s)^{q-1} E_{q, q}\left((-d+a)(t-s)^{q}\right)\left(12 a R+4 \omega_{R}(s)+2\right) d s+ \\
+4 R \int_{0}^{t}(t-s)^{q-1} E_{q, q}\left((-d+a)(t-s)^{q}\right)\left\|f^{2}(t)-g_{\varepsilon_{k}}^{2}(t)\right\| d s .
\end{gathered}
$$

Notice that the second, third, and fourth terms tend to zero as $k \rightarrow \infty$. In fact, in the third term, the integral is uniformly bounded on $[0, T]$, and we can apply Remark 3 to the second and fourth terms.

Passing in (39) to the limit as $k \rightarrow \infty$, we get

$$
\left\|x^{1}(t)-x^{2}(t)\right\|^{2} \leq 0 .
$$

Therefore, for each $t \in[0, T]$, it holds that $x^{1}(t)=x^{2}(t)$.

\section{An Example}

Consider the following Cauchy problem for a system governed by a partial differential inclusion of a fractional diffusion type:

$$
\begin{gathered}
\partial_{t}^{q} u(t, s) \in \frac{\partial^{2} u}{\partial s^{2}}(t, s)-\mu u(t, s)+f(t, s, u(t, s))+\psi(t, s)+D(u(t, \cdot))(s), \\
u(0, s)=\gamma(s),
\end{gathered}
$$


where $t \in[0, T], s \in(-\infty,+\infty), \mu>0, \partial_{t}^{q}$ is the Caputo partial derivative in $t$ of order $0<$ $q<1, f:[0, T] \times(-\infty,+\infty) \times \mathbb{R} \rightarrow \mathbb{R}, \psi \in L^{2}([0, T] \times(-\infty,+\infty)), D: L^{2}(-\infty,+\infty) \rightarrow$ $K v\left(L^{2}(-\infty,+\infty)\right)$ is the feedback multimap which will be defined below, and $D(x)(s)=$ $\{\omega(s): \omega \in D(x)\}$.

Considering $u(t, s)$ as $x(t)(s)$, where $x:[0, T] \rightarrow L^{2}(-\infty,+\infty)$, we will reduce the above problem to abstract problems (15) and (16) in the space $H:=L^{2}(-\infty,+\infty)$. In so doing, the operator $A$ is defined by the formula

$$
A x=\frac{\partial^{2} x}{\partial s^{2}} .
$$

We will assume that the function $f$ generates the superposition operator $\phi:[0, T] \times H \rightarrow H$ defined as

$$
\phi(t, x)(s)=f(t, s, x(s)) .
$$

In order to conclude that this operator is well defined, it is sufficient to assume that the function $f$ is continuous, $f(t, \cdot, v) \in H$ for all $(t, v) \in[0, T] \times \mathbb{R}$, and $f$ has a sublinear growth in the third variable:

$$
|f(t, s, v)| \leq a+b|v|, \quad \forall(t, s) \in[0, T] \times(-\infty,+\infty),
$$

where $a$ and $b$ are some nonnegative constants.

We now describe the "feedback" multimap $D$. For a given concave locally Lipschitzfunctional

$$
g: \mathbb{R}^{n} \rightarrow \mathbb{R},
$$

we denote by $\partial g$ its subdifferential. It is known (see [40], Propositions 2.1.2, 2.1.5, and 2.1.9) that $\partial g$ is a u.s.c. multimap in $\mathbb{R}^{n}$ with compact convex values, which is monotone in the following sense:

$$
\langle\widetilde{X}-\widetilde{Y}, X-Y\rangle_{\mathbb{R}^{n}} \leq 0
$$

for all $X, Y \in \mathbb{R}^{n} ; \widetilde{X} \in \partial g(X), \widetilde{Y} \in \partial g(Y)$.

Now, let $\left\{\varphi_{i}\right\}_{i=1}^{n}$ be any fixed orthonormal system of functions from $H$. For a given $x \in H$, we define a vector $X(x) \in \mathbb{R}^{n}$ assuming $X(x)=\left\{\kappa_{1}, \ldots, \kappa_{n}\right\}$, where

$$
\kappa_{i}=\left\langle x, \varphi_{i}\right\rangle_{H}, \quad i=1, \ldots, n .
$$

We now define the multioperator $D: H \rightarrow P(H)$ as

$$
D(x)=\left\{\sum_{i=1}^{n} \zeta_{i} \varphi_{i}:\left(\zeta_{1}, \ldots, \zeta_{n}\right) \in \partial g(X(x))\right\} .
$$

From the properties of multivalued maps (see, e.g., [33]), it follows that $D$ is u.s.c. and has compact convex values; moreover, from (43)-(45), it follows that $D$ is monotone, i.e.,

$$
\langle\widetilde{x}-\widetilde{y}, x-y\rangle_{H} \leq 0
$$

for all $x, y \in H, \widetilde{x} \in D(x), \widetilde{y} \in D(y)$.

Now, we can substitute problems (41) and (42) with the following problem in the space $H$ :

$$
\begin{gathered}
D_{0}^{q} x(t) \in A x(t)-\mu x(t)+\phi(t, x(t))+\widetilde{\psi}(t)+D(x(t)), \\
x(0)=\gamma \in H,
\end{gathered}
$$

where $\widetilde{\psi}(t)(s)=\psi(t, s)$. 
If we suppose that $f(t, s, v)$ is continuously differentiable in $v,\left|\frac{\partial f}{\partial v}(t, s, v)\right|$ is bounded for all $t \in[0, T], s \in(-\infty,+\infty)$, and

$$
\frac{\partial f}{\partial v}(t, s, v)<-\alpha \text { for some } \alpha>0
$$

then

$$
\langle\phi(t, x)-\phi(t, y), x-y\rangle_{H} \leq-\alpha\|x-y\|_{H}^{2}, \quad \forall x, y \in H .
$$

From the properties of u.s.c. compact-valued maps (see [33], Theorem 1.2.35), it follows that $D$ transforms bounded subsets of $H$ into relatively compact ones. However, then,

$$
\chi_{H}(\phi(t, \Omega)+D(\Omega)) \leq K \chi_{H}(\Omega),
$$

where $K=\sup \left\{\left|\frac{\partial f}{\partial v}(t, s, v)\right|: t \in[0, T], s \in(-\infty,+\infty), v \in \mathbb{R}\right\}$.

So, all conditions of Theorems 2 and 3 are fulfilled, and we conclude that problems (41) and (42) have a unique mild solution.

\section{Conclusions}

We studied the Cauchy problem for a semi-linear differential inclusion of a fractional order in a Hilbert space. The existence and uniqueness of a mild solution to this problem are obtained under assumptions that the linear part of the inclusion is a linear monotone operator generating a $C_{0}$-semigroup, whereas the multivalued part satisfies a certain monotonicity-type condition. An example concerning the existence and uniqueness of a mild solution for a system governed by a partial fractional-order differential inclusion is presented. As was shown in the paper [13], the uniqueness of a solution entails a difference from zero of its topological index. In the forthcoming research, by using the results from [13], this will allow the substantiation of a semi-discretization scheme for obtaining approximate solutions to the Cauchy problem for differential inclusions of fractional order.

Author Contributions: Conceptualization, M.K., V.O. and J.-C.Y.; methodology, M.K., V.O. and J.-C.Y.; software, G.P.; validation, M.K., G.P. and J.-C.Y.; formal analysis, V.O., G.P. and J.-C.Y.; investigation, M.K., G.P. and J.-C.Y.; writing—original draft preparation, V.O. and G.P.; writing-review and editing, V.O. and G.P.; supervision, J.-C.Y.; project administration, J.-C.Y.; funding acquisition, V.O. and J.-C.Y. All authors have read and agreed to the published version of the manuscript

Funding: The work of the first, second, and the third authors was supported by the State contract of the Russian Ministry of Science and Higher Education as part of the state task (contract FZGF-20200009). The work of the fourth author was supported by the MOST grant number 108-2115-M-039 $-005-\mathrm{MY} 3$.

Institutional Review Board Statement: Not applicable.

Informed Consent Statement: Not applicable.

Data Availability Statement: Not applicable.

Conflicts of Interest: The authors declare no conflict of interest.

\section{References}

1. Kilbas, A.A.; Srivastava, H.M.; Trujillo, J.J. Theory and Applications of Fractional Differential Equations; North-Holland Mathematics Studies; Elsevier Science B.V.: Amsterdam, The Netherlands, 2006.

2. Podlubny, I. Fractional Differential Equations; Academic Press: San Diego, CA, USA, 1999.

3. Zhou, Y. Fractional Evolution Equations and Inclusions: Analysis and Control; Elsevier Academic Press: London, UK, 2016.

4. Afanasova, M.; Liou, Y.C.; Obukhoskii, V.; Petrosyan, G. On controllability for a system governed by a fractional-order semilinear functional differential inclusion in a Banach space. J. Nonlinear Convex Anal. 2019, 20, 1919-1935.

5. Afanasova, M.; Petrosyan, G. On the boundary value problem for functional differential inclusion of fractional order with general initial condition in a Banach space. Russ. Math. 2019, 63, 1-12. [CrossRef]

6. Agarwal, R.P.; Ahmad, B. Existence theory for anti-periodic boundary value problems of fractional differential equations and inclusions. Comput. Math. Appl. 2011, 62, 1200-1214. [CrossRef] 
7. Appell, J.; Lopez, B.; Sadarangani, K. Existence and uniqueness of solutions for a nonlinear fractional initial value problem involving Caputo derivatives. J. Nonlinear Var. Anal. 2018, 2, 25-33.

8. Benedetti, I.; Obukhovskii, V.; Taddei, V. On generalized boundary value problems for a class of fractional differential inclusions. Fract. Calc. Appl. Anal. 2017, 20, 1424-1446. [CrossRef]

9. Mainardi, F.; Rionero, S.; Ruggeri, T. On the initial value problem for the fractional diffusion-wave equation. In Waves and Stability in Continuous Media; World Scientific: Singapore, 1994; pp. 246-251.

10. Kamenskii, M.; Obukhovskii, V.; Petrosyan, G.; Yao, J.C. On semilinear fractional order differential inclusions in Banach spaces. Fixed Point Theory 2017, 18, 269-292. [CrossRef]

11. Kamenskii, M.; Obukhovskii, V.; Petrosyan, G.; Yao, J.C. Boundary value problems for semilinear differential inclusions of fractional order in a Banach space. Appl. Anal. 2018, 97, 571-591. [CrossRef]

12. Kamenskii, M.; Obukhovskii, V.; Petrosyan, G.; Yao, J.C. On approximate solutions for a class of semilinear fractional-order differential equations in Banach spaces. Fixed Point Theory Appl. 2017, 28, 1-28. [CrossRef]

13. Kamenskii, M.; Obukhovskii, V.; Petrosyan, G.; Yao, J.C. Existence and Approximation of Solutions to Nonlocal Boundary Value Problems for Fractional Differential Inclusions. Fixed Point Theory Appl. 2019, 2019, 1-21. [CrossRef]

14. Kamenskii, M.; Obukhovskii, V.; Petrosyan, G.; Yao, J.C. On a Periodic Boundary Value Problem for a Fractional Order Semilinear Functional Differential Inclusions in a Banach Space. Mathematics 2019, 7, 1146. [CrossRef]

15. Ke, T.D.; Loi, N.V.; Obukhovskii, V. Decay solutions for a class of fractional differential variational inequalities. Fract. Calc. Appl. Anal. 2015, 18, 531-553.

16. Ke, T.D.; Obukhovskii, V.; Wong, N.C.; Yao, J.C. On a class of fractional order differential inclusions with infinite delays. Appl. Anal. 2013, 92, 115-137. [CrossRef]

17. Obukhovskii, V.; Zecca, P.; Afanasova, M. Boundary value problems for fractional-order differential inclusions in Banach spaces with nondensely defined operators. Fixed Point Theory 2021, 22, to appear.

18. Petrosyan, G. On antiperiodic boundary value problem for a semilinear differential inclusion of fractional order with a deviating argument in a Banach space. Ufa Math. J. 2020, 12, 69-80. [CrossRef]

19. Petrosyan, G.; Afanasova, M. On the Cauchy problem for differential inclusion of fractional order with a nonlinear boundary condition. Bull. Voronezh State Univ. Ser. Phys. Maths. 2017, 1, 135-151. (In Russian)

20. Coddington, E.A.; Levinson, N. Theory of Ordinary Differential Equations; McGraw-Hill Book Company, Inc.: New York, NY, USA; Toronto, AB, Canada; London, UK, 1955.

21. Hartman, P. Ordinary Differential Equations; John Wiley and Sons: New York, NY, USA; London, UK; Sydney, Australia, 1964.

22. Krasnoselskii, M.A. The Operator of Translation along the Trajectories of Differential Equations. Translation of Mathematical Monographs, 19; American Mathematical Society: Providence, RI, USA, 1968.

23. Brezis, H.; Pazy, A. Accretive sets and differential equations in Banach spaces. Israel J. Math. 1970, 8, 367-383. [CrossRef]

24. Perov, A.I.; Trubnikov, Y.V. Monotonic equations I. Differ. Equ. 1975, 10, 619-627.

25. Aguila-Camacho, N.; Duarte-Mermoud, M.A.; Gallegos, J.A. Lyapunov functions for fractional order systems. Commun. Nonlinear Sci. Numer. Simul. 2014, 19, 2951-2957. [CrossRef]

26. Gomoyunov, M.I. Fractional derivatives of convex Lyapunov functions and control problems in fractional order systems. Fract. Calc. Appl. Anal. 2018, 21, 1238-1261. [CrossRef]

27. Gomoyunov, M.I. Approximation of fractional order conflict-controlled systems. Prog. Fract. Differ. Appl. 2019, 5, 143-155. [CrossRef]

28. Gorenflo, R.; Kilbas, A.A.; Mainardi, F.; Rogosin, S.V. Mittag-Leffler Functions, Related Topics and Applications; Springer-Verlag: Berlin/Heidelberg, Germany, 2014.

29. Srivastava, H.M.; Bansal, D. Close-to-convexity of a certain family of q-Mittag-Leffler functions. J. Nonlinear Var. Anal. 2017, 1, 61-69.

30. Wang, R.-N.; Chen, D.-H.; Xiao, T.-J. Abstract fractional Cauchy problems with almost sectorial operators. J. Differ. Equ. 2012, 252, 202-235. [CrossRef]

31. Zhang, Z.; Liu, B. Existence of mild solutions for fractional evolution equations. Fixed Point Theory 2014, 15, 325-334.

32. Kamenskii, M.; Obukhovskii, V.; Zecca, P. Condensing Multivalued Maps and Semilinear Differential Inclusions in Banach Spaces; Walter de Gruyter: Berlin, Germany; New York, NY, USA, 2001.

33. Obukhovskii, V.; Gel'man, B. Multivalued Maps and Differential Inclusions. Elements of Theory and Applications; World Scientific Publishing Co.: Hackensack, NJ, USA, 2020.

34. Getmanova, E.; Obukhovskii, V.; Yao, J.-C. A random topological fixed point index for a class of multivalued maps. Appl. Set-Valued Anal. Optim. 2019, 1, 95-103.

35. Getmanova, E.; Obukhovskii, V. A note on random equilibrium points of two multivalued maps. J. Nonlinear Var. Anal. 2018, 2, 269-272.

36. Kamenskii, M.; Voskovskaya, N.; Zvereva, M. On periodic oscillations of some points of a string with a nonlinear boundary condition. Appl. Set-Valued Anal. Optim. 2020, 2, 35-48.

37. Yosida, K. Functional Analysis; Springer-Verlag: Berlin/Heidelberg, Germany; New York, NY. USA, 1980.

38. Engel, K.-J.; Nagel, R. A Short Course on Operator Semigroups; Springer-Verlag: New York, NY, USA, 2006. 
39. Bogdan, V.M. Generalized vectorial Lebesgue and Bochner integration theory. arXiv 2010, arXiv:1006.3881.

40. Clarke, F.H. Optimization and Nonsmooth Analysis, 2nd ed.; Classics in Applied Mathematics; Society for Industrial and Applied Mathematics (SIAM): Philadelphia, PA, USA, 1990. 